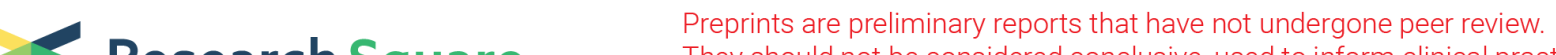 (5) or referenced by the media as validated information.
}

\section{Multiplex and On-site PCR detection of swine diseases based on the microfluidic chip system}

\section{Yan Jiang}

Nanjing Customes

Shan Jiang

Nanjing,Customs

Yue Wu

Nanjing Agricultural University

Bin Zhou ( $\nabla$ zhoubin@njau.edu.cn )

Nanjing Agricultural University https://orcid.org/0000-0001-7279-9489

Kaiming Wang

Nanjing,Customs

Luyan Jiang

Nanjing Customs

Yunfeng Long

Nanjing Tech University

\section{Gan Chen}

Jiangganshan Agricultural Science and Technology Park Management Committee

Dexin Zeng

Nanjing,Customs

\section{Methodology article}

Keywords: swine disease, microfluidic chips, PCR, multiplex and on-site detection

Posted Date: September 11th, 2020

DOI: https://doi.org/10.21203/rs.2.21078/v5

License: (c) (1) This work is licensed under a Creative Commons Attribution 4.0 International License. Read Full License

Version of Record: A version of this preprint was published on March 12th, 2021. See the published version at https://doi.org/10.1186/s12917-021-02825-w. 


\section{Abstract}

Background》 At present, the process of inspection and quarantine starts with sampling at the customs port, continues with transporting the samples to the central laboratory for inspection experiments, and ends with the inspected results being fed back to the port. This process had the risks of degradation of biological samples and generation of pathogenic microorganisms and did not meet the rapid on-site detection demand because it took a rather long time. Therefore, it is urgently needed to develop a rapid and high-throughput detection assay of pathogenic microorganisms at the customs port. The aim of this study was to develop a microfluidic chip to rapidly detect swine pathogenic microorganisms with highthroughput and higher accuracy. Moreover, this chip will decrease the risk of spreading infection during transportation.

Results: A series of experiments were performed to establish a microfluidic chip. The resulting data showed that the positive nucleic acid of four swine viruses were detected by using a portable and rapid microfluidic PCR system, which could achieve a on-site real-time quantitative PCR detection. Furthermore, the detection results of eight clinical samples were obtained within an hour. The detection limit of this microfluidic PCR detection system was as low as 1 copies $/ \mu \mathrm{L}$. The results showed that the high sensitivity and specificity of this chip system in disease detection played an important role in customs inspection and quarantine during customs clearance.

Conclusion: The microfluidic PCR detection system established in this study could meet the requirement for rapid detection of samples at the customs port. This chip could avoid the risky process of transporting the samples from the sampling site to the testing lab, and drastically reduce the inspection cycle. Moreover, it would enable parallel inspections on one chip, which greatly raised the efficiency of inspection.

\section{Background}

Polymerase chain reaction (PCR) is a molecular detection technology based on nucleic acid sequences [1]. After nearly 40 years development, the PCR molecular detection technology has become the primary method for detecting diseases caused by pathogenic microorganisms, and has been widely used in disease detection [2,3], clinical testing [4,5], food quarantine [6,7], forensic identification [8,9], customs inspection [10] and in various other fields [11-14]. The traditional PCR instrument is large in size and inconvenient to carry; and it has a higher demand for the experimental environmen. These drawbacks of this instrument seriously limit its scope of use, and prevent its operation in rapid on-site real-time PCR detection [15].

Porcine circovirus (PCV) belongs to the Circoviridae family, and is one of the smallest known animal viruses. The virion has no envelope, and contains a covalently closed single-strand circular negativestrand DNA. It often causes weaning piglets with multiple system failure syndrome (PMWS) and was tested using quantitative PCR and antigen capture ELISA [16]. Porcine reproductive and respiratory 
syndrome (PRRSV) is a member of the Arteritis virus family. According to the antigenicity, genome and pathogenicity of the virus, PRRSV can be divided into two types, namely European type and American type. It mainly causes reproductive disorders in pigs and first isolated in China in 1996[17]. Porcine epidemic diarrhea virus (PEDV) is a single-positive-strand RNA virus, belonging to the Coronavirus. PEDV is one of the major etiologies responsible for the acute, highly contagious disease in the digestive tract of pigs, especially neonatal piglets. Multiplex PCR is commonly used in clinical practice to differentiate between this virus, rotavirus and porcine epidemic diarrhea [18]. Pseudorabies virus (PRV) can cause reproductive disorders at any stage of pregnancy, piglets often show chin and respiratory symptoms. Some researchers have designed primers for $\mathrm{gE}$ and $\mathrm{gB}$ genes to establish gene chip detection methods to distinguish between field and vaccine virus [19].

The microfluidic chip [20-22], also known as lab on a chip, combines and arranges various unit technologies on a small platform to implement all the biochemical laboratory experiments including sampling, sample introduction, sample preparation, reaction, product testing, and so on [23,24]. Compared with the traditional PCR method, the microfluidic chip is much smaller in size and more portable along with much less reagents. It, changed temperature more rapidly, integrated with other devices more easily, and had more moderate demands for experimental environment and lab assistants[25-28]. All these advantages brought the microfluidic chip into the focus of research on rapid on-site real-time PCR detection [29-32]. Pork and its processed products, as a traditional food, have become an indispensable part in people's diet [33]. China Customs, as the only pass for import and export of goods and commodities into and out of the country, is responsible for the inspection and quarantine of tens of thousands of pigs, and large amounts of pork and its processed products; which calls for rapid, efficient and safe work[34]. The current detection methods cannot meet the needs of rapid detection [3536] . Therefore, development of a technology for rapid and high-throughput detection of pathogenic microorganisms at the customs port is of great significance. This study was to develop a microfluidic chip to be applied to rapid and high-throughput swine disease detection with higher accuracy and lower risk of spreading pathogenic microorganisms during transportation.

\section{Results}

\section{The limit of detection test using standard RNA and DNA samples.}

We choose HCV virus RNA and HBV virus DNA standard sample as a reference for LOD evaluation. The HCV RNA and HBV DNA standard samples are diluted to a concentration of 2000 copies $/ \mathrm{ml}, 1000$ copies $/ \mathrm{ml}$ and 500 copies $/ \mathrm{ml}$. At each concentration, 30 sample replicates were prepared for a probit analysis. We apply $95 \%$ sensitivity as a threshold for a detection limit determination and a $95 \%$ confidence interval is calculated as well. The results are shown in Table 2 and we could conclude that the detection limit for HCV RNA sample is around 1000 2000 copies/ml and that of HBV DNA sample is 1000 copies/ml. 
The detection limit testing using plasmid sample. We then chose four principal swine viruses, including PRRSV, PEDV, PRV and PCV2, which are often found in related inspection at the customs, and we synthesized the plasmids of these viruses as positive references. The concentrations of the primers and probes, and the reaction temperatures were optimized by means of the plasmid amplifications on the PCR microfluidic chips. The microfluidic chip had 8 chambers, and each chamber accommodate $2 \mu \mathrm{L}$ of the PCR reaction solution. The volume of the amplification system was reduced that was helpful in the rapid PCR process. The rapid heat transfer kept the activity of the Taq DNA polymerase at the maximum level during the rise and fall of temperature. The microfluidic chip with silicon as its substrate further accelerated the heat transfer during the PCR thermal cycle, because of silicon's excellent thermal conductivity. It was obvious that the amplification reaction's fluorescence intensity on the chip presented itself in a typical S-shaped curve, and the negative control chamber, in which sterilized water was used as the template, did not show any amplification, indicating that the specificity of the primers and the probe, and the optimization of the amplification temperature were all appropriate here.

The initial concentration of the four plasmids was $10^{9}$ copies $/ \mu \mathrm{L}$. All the plasmids were 10 -fold serial diluted to the final concentration of 1 copies $/ \mu \mathrm{L}$, and every concentration was tested. As shown in Fig 2 , the sensitivity test of the four positive plasmids were $10 \mathrm{copies} / \mu \mathrm{L}$ for PRRSV, 10 copies/ $\mu \mathrm{L}$ for PEDV, 100 copies/ $\mu \mathrm{L}$ for PRV and 1 copy/ $\mu \mathrm{L}$ for PCV2, respectively, which would meet the requirement for detection in terms of the virus load obtained from the clinical samples of the animals.

The specificity testing using positive nucleic acid. Besides the sensitivity test, we also verified the specificity performance of the PCR detections on the microfluidic chip. Based on the positive nucleic acids of four viruses isolated from the clinical sick pigs, which performed the typical symptoms of reproductive disorders, multi-system failure of weaned piglets, and watery diarrhea. We chose one sample as the target for the specificity test, and the other three samples were used as the control. The PCR amplifications were performed on one chip with one reaction chamber filled with the target sample, the rest of the chambers filled with the other three samples as well as other two porcine-transmitted viral agents including porcine transmissible gastroenteritis virus (TGEV) and procine rotavirus (PRVA). As shown in Fig 3, the result showed that the four PCR protocols all had a very high specificity, because only the target sample presented a significant amplification curve and the control samples had no amplifications. The fast amplification process of the PCR system contributed not only to the high specificity of the PCR amplification but also the short duration in the non-required temperature zone.

The detection performance of the microfluidic PCR system using positive nucleic acid. Finally, we verified the PCR microfluidic chip detection performance using the positive nucleic acid of swine pathogens. Every two chambers on the chip were used for the reaction of one clinical sample, and one chip accommodated four samples in parallel reactions. At the same time, we also performed the detection for the viruses of four swine diseases on a traditional tube-based PCR instrument for parallel reference. As shown in Fig. 4A, each of the four samples had a significant $S$ curve with a varied Ct value (17.5 for PRRSV, 33.5 for PEDV, 18.5 for PRV and 16.5 for PCV2, respectively). The Ct values of the four swine diseases tested on chip-based and tube-based PCR platform showed no significant differences (Fig.4 B). 
The differences in the Ct values were due to the variety in the virus abundance of each of the original positive nucleic acid. The magnitude of the fluorescence intensity increment in the different positive nucleic acid at the end of the reaction was due to the different amplification efficiency of primers and probes for different target gene sequences. However, the Ct value was usually used as a criterion when determining the positive or negative nature of the sample. The sample with a Ct value smaller than 35 could be considered as positive threshold, and the sample with a Ct value greater than 35 needed to be reexamined.

\section{Discussion}

The aim of this study is to develop a fast and on-site swine disease detection solutions to decrease the transit time for customs clearance. Point of care testing could be the optimal choice for the on-site detection. Compared with molecule hybridization [36], LAMP [37] and other isothermal amplification technology, PCR technology as the gold standard for infectious disease detection which has been widely applied However, the current PCR performance still need sample transport to testing lab and the resulting data could not been got in less than $3 \mathrm{~h}$ [38]. The challenge is that the sample have a risk of degradation if the storage condition is not proper as well as extra cost from the transportation. Therefore, a portable and miniaturized PCR detection platform is needed to develop. Herein, the microfluidics chip based PCR testing solution along with short testing duration, multiple testing capability and instrument portability was established for diagnostic assay.

Compared to traditional lab-based PCR detection that must be finished in the central lab, our portable PCR tester could realize an on-site detection for swine diseases and a rapid result observation. In addition, four swine pathogens could be detected on a single chip simultaneously. A rapid thermal cycling capability of the PCR tester with the help of microheater realized a shorter detection time, which is helpful for a fast custom clearance and also for an efficient disease control to decrease the further spread risk of infection disease. In order to realize an on-site molecular test, many factors need to be considered, including testing duration, instrument portability, operation complication, contamination and so on. A real time PCR method may avoid the contamination of this process and need no more offline analysis because of. a miniaturization of the optical detection module for a fluorescent detection. Around 110 copies/uL detection limit on chip could meet a fast screening demand and has a fine accordance with that of the traditional PCR instrument. As for the specificity of this method, no cross reaction occurred when more than one PCR detection performed in a single chip well, suggesting that the PCR reagent component had a good compatibility with the chip surface. Additionally, a fast thermal cycling could increase the PCR enzyme amplification efficiency. Therefore, this chip is helpful for the further development of a multiple optical channel instrument for high-throughput multiplex PCR detection. In the future, a more high-throughput chip that could realize a more than 16 samples testing on a single chip will be developed, which could accommodate more samples for simultaneous detection, along with higher throughput, greater efficiency and accuracy.The further study also needs to develop an automated sample preparation system for nucleic acid extraction and purification, which will decrease the on-thespot detection duration [39-41]. 


\section{Conclusions}

In this study, An on-site microfluidic PCR detection system established meeted the requirement for rapid detection of samples at the customs port. All the detection were completed within $1 \mathrm{~h}$. Application of this chip would effectively help the on-site inspection to be carried out and it could avoid the risky process of transporting the samples from the sampling site to the testing lab and drastically reduce the inspection cycle.

\section{Methods}

Reagents and equipment. The magnetic bead virus nucleic acid extraction kit was purchased from Suzhou Tianlong Bio-Technology Co. Ltd. The reverse transcriptase was from Vazyme Biotech Co. Ltd. (Nanjing), and the Taq DNA polymerase was from Fapon Biotech Inc. (Zhuhai). The primers and probes used in common epidemic swine diseases detection were synthesized by Genscript (Nanjing) Co., Ltd, and the detailed sequences are shown in Appendix Table 1. The microfluidic PCR analyzer was purchased from Shenzhen Shineway Technology Corporation and the fluorescence quantitative PCR instrument was from Hangzhou Bioer Technology Co. Ltd.

Nucleic acid extraction and amplification. The nucleic acid of the pathogenic microorganisms were extracted following the instructions of the magnetic bead nucleic acid extraction kit, and the extracted DNA solution was used as the template solution for the subsequent experiments, and was stored at $-20^{\circ}$ $\mathrm{C}$ in a refrigerator; or at $4^{\circ} \mathrm{C}$ in a refrigerator or on ice in case of immediate use in a subsequent experiment. In the nucleic acid amplification, a $25 \mu$ Lreaction solution was prepared with the following components: $5 \mu$ Ltemplate solution, $0.5 \mu$ Lforward primer $(10 \mu \mathrm{M}), 0.5 \mu$ Lreverse primer $(10 \mu \mathrm{M}), 0.2$ $\mu \mathrm{Lprobe}(10 \mu \mathrm{M}), 0.2 \mu \mathrm{Lreverse}$ transcriptase, $0.5 \mu \mathrm{LTaq}$ DNA polymerase, $5 \mu \mathrm{L} 5 \mathrm{X}$ reaction buffer, and 13.1 $\mu$ Lsterilized water. All these components were fully mixed in a PCR tube and added to the microfluidic chip by a pipette, then PCR amplification was performed. The process of the amplification included Step One: reverse transcription at $50^{\circ} \mathrm{C}$ for 15 minutes; Step Two: denaturation at $95^{\circ} \mathrm{C}$ for 3 minutes; and Step Three: 40 cycles at $95^{\circ} \mathrm{C}$ for 15 seconds and at $55^{\circ} \mathrm{C}$ for 45 seconds.

Chip design and preparation. There were 8 reaction chambers with a volume of $2 \mu \mathrm{L}$ each on the PCR microfluidic chip. The PCR microfluidic chips were made of silicon wafers of $500 \mu \mathrm{m}$ thick with both sides polished and heat-resistant glass substrates of $500 \mu \mathrm{m}$ thick by Shenzhen Shineway Technology Corporation. The PCR reaction chambers and channels on the silicon wafers were formed by dry etching using a semiconductor photolithography process. Then, sixteen holes were drilled on the silicon substrate which served as the inlets and outlets of PCR solutions, respectively. Finally, the silicon wafers were bonded to the glass substrates and the two-layer wafers were cut by a dicing saw to form independent PCR detection chips.

a standardized semiconductor fabrication process was utilized to ensure the yield rate and uniformity in quality of the chips. And the automated fabrication process could significantly reduce the cost of each 
single inspection by expanding the size of the silicon wafers.

The amplification detection system of the PCR microfluidic chip. A portable rapid microfluidic PCR system (SWA-01, Shenzhen Shineway Technology Corporation) was used to perform the real-time PCR amplification of the samples (Fig. 1). The dimension was $330 \mathrm{~mm} \nabla \mathrm{L} \nabla \times 320 \mathrm{~mm} \nabla \mathrm{W} \nabla \times 160 \mathrm{~mm} \nabla \mathrm{H} \backslash$ and the weight was round $3 \mathrm{~kg}$, suggesting it had a small footprint. Used in conjunction with the specially supplied reagents and the microfluidic chips, the SWA-01 PCR instrument is applicable to rapid real-time fluorescence detection of PCR in the fields of animal quarantine, food safety and public health and safety. The portable rapid microfluidic PCR instrument was mainly composed of a control system (with a touch screen graphic user interface), a power supply system, a photoelectric system, a temperature control system [35], an outer jacket part, a microfluidic chip, and a software module. The instrument had the following advantages for on-site detection as follows: portable design, low weight, rapid thermal cycling and real time detection.

\section{Abbreviations}

PCR: Polymerase chain reaction; ELISA:Enzyme-linked immunosorbent assay; LAMP:Loop-mediated isothermal amplification

\section{Declarations}

\section{Ethics approval and consent to participate}

Not applicable.

\section{Consent for publication}

Not applicable Competing interest.

\section{Availability of data and materials}

Raw data is available from the corresponding author on reasonable request.

\section{Competing Interests}

The authors declare that they have no competing interests.

Funding: This work and the writing of the manuscript were financially supported by the National Key Research and Development Program of China (2016YFD050110402). The funder had no role in the design of the collection, analysis, and interpretation of data.

\section{Authors' contributions}


YJ designed and directed the study. SJ,LYJ,YFL and YJ perform the experiment, collect results and analyze data. YW and BZ draft the first vision of the manuscript. KMW, GC and DXZ collect clinical serum samples. BZ critically revised the manuscripts. All the participants carefully reviewed this vision manuscript.All authors have read and approved the manuscript.

Acknowledgments: we give our acknowledgment to Shenzhen Shineway Technology

Corporation for their support of chip design and fabrication and microchip based PCR detection instrument.

\section{Author details}

1 Animal ,Plant and Food Inspection Center, Nanjing Customs;

2 MOE Joint International Research Laboratory of Animal Health and Food Safety, College of Veterinary Medicine, Nanjing Agricultural University, Nanjing 210095, China

3 Jinggangshan Agricultural Science and Technology Park Management Committee

\section{Publisher's Note}

Springer Nature remains neutral with regard to jurisdictional claims in published maps and institutional affiliations.

\section{References}

1. Saiki, R.K.; Scharf, S.; Faloona, F.; Mullis, K.B.; Horn, G.T.; Erlich, H.A.; Arnheim, N. Enzymatic amplification of beta-globin genomic sequences and restriction site analysis for diagnosis of sickle cell anemia. Science 1985, 230, 1350-1354.

2. Watanabe, M.; Kawaguchi, T.; Isa, S.; Ando, M.; Tamiya, A.; Kubo, A.; Saka, H.; Takeo, S.; Adachi, H.; Tagawa, T.; et al. Ultra-Sensitive Detection of the Pretreatment EGFR T790M Mutation in Non-Small Cell Lung Cancer Patients with an EGFR-Activating Mutation Using Droplet Digital PCR. Clin. Cancer Res. 2015, 21, 3552-3560.

3. Pekin, D.; Skhiri, Y.; Baret, J.-C.; Corre, D.L.; Mazutis, L.; Salem, C.B.; Millot, F.; Harrak, A.E.; Hutchison, J.B.; Larson, J.W.; et al. Quantitative and sensitive detection of rare mutations using droplet-based microfluidics. Lab Chip 2011, 11, 2156-2166.

4. Mo, Q.H.; Yang, C.L.; Lin, J.C.; Tan, H.; Tu, C.N.; Ye, L.Q.; Liu, Z.M.; Du, J.; Sun, H.; Yang, Z. [One-step multiplex RT-PCR for rapid screening of type A, B and novel A (H1N1) influenza viruses]. Nan Fang Yi Ke Da Xue Xue Bao 2009, 29, 1545-1547.

5. Sippert, E.; Rocha, B.C.; Assis, F.L.; Ok, S.; Rios, M. Use of Monocyte-Derived Macrophage Culture Increases Zika Virus Isolation Rate from Human Plasma. Viruses 2019, 11, 1058. 
6. Morisset, D.; Štebih, D.; Milavec, M.; Gruden, K.; Žel, J. Quantitative Analysis of Food and Feed Samples with Droplet Digital PCR. PLOS ONE 2013, 8, e62583.

7. Clayton, E.M.; Hill, C.; Cotter, P.D.; Ross, R.P. Real-Time PCR Assay To Differentiate Listeriolysin SPositive and -Negative Strains of Listeria monocytogenes. Appl. Environ. Microbiol. 2011, 77, 163171.

8. Jung, J.Y.; Yoon, H.K.; An, S.; Lee, J.W.; Ahn, E.-R.; Kim, Y.-J.; Park, H.-C.; Lee, K.; Hwang, J.H.; Lim, S.-K. Rapid oral bacteria detection based on real-time PCR for the forensic identification of saliva. Sci. Rep. 2018, 8, 1-10.

9. Sauer, E.; Reinke, A.-K.; Courts, C. Differentiation of five body fluids from forensic samples by expression analysis of four microRNAs using quantitative PCR. Forensic Sci. Int.: Gen. 2016, 22, 8999.

10. Han, Y.; Gu, Q.; Lv, D.; Wang, S.; Zhang, L. Risk Analysis on Carp Edema Virus being Released into China. China Animal Health Inspection 2018, 7.

11. Ogawa, H.; Taira, O.; Hirai, T.; Takeuchi, H.; Nagao, A.; Ishikawa, Y.; Tuchiya, K.; Nunoya, T.; Ueda, S. Multiplex PCR and multiplex RT-PCR for inclusive detection of major swine DNA and RNA viruses in pigs with multiple infections. J. Virol. Methods 2009, 160, 210-214.

12. Blakely, R.D.; Berson, H.E.; al, et Cloning and Expression of a Functional Serotonin Transporter from Rat Brain. Nature; London 1991, 354, 66-70.

13. Takeuchi, S.; Itoh, N.; Uchio, E.; Aoki, K.; Ohno, S. Serotyping of Adenoviruses on Conjunctival Scrapings by PCR and Sequence Analysis. J. Clin. Microbiol. 1999, 37, 1839-1845.

14. Pan, W.; Chen, W.; Jiang, X. Microfluidic Western Blot. Anal. Chem. 2010, 82, 3974-3976.

15. McKillen, J.; Hjertner, B.; Millar, A.; McNeilly, F.; Belák, S.; Adair, B.; Allan, G. Molecular beacon real-time PCR detection of swine viruses. J. Virol. Methods 2007, 140, 155-165.

16. McNeilly F, McNair I, O'Connor M, Brockbank S, Gilpin D, Lasagna C, Boriosi G, Meehan B, Ellis J, Krakowka $S$ et al: Evaluation of a porcine circovirus type 2-specific antigen-capture enzyme-linked immunosorbent assay for the diagnosis of postweaning multisystemic wasting syndrome in pigs: comparison with virus isolation, immunohistochemistry, and the polymerase chain reaction. $J$ Vet Diagn Invest 2002, 14(2), 106-112.

17. Duinhof TF, van Schaik G, van Esch EJ, Wellenberg GJ: Detection of PRRSV circulation in herds without clinical signs of PRRS: comparison of five age groups to assess the preferred age group and sample size. Vet Microbio/ 2011, 150(1-2), 180-184.

18. Masuda T, Tsuchiaka S, Ashiba T, Yamasato H, Fukunari K, Omatsu T, Furuya T, Shirai J, Mizutani T, Nagai M: Development of one-step real-time reverse transcriptase-PCR-based assays for the rapid and simultaneous detection of four viruses causing porcine diarrhea. Jpn J Vet Res 2016, 64(1),514.

19. Huang J, Ma G, Fu L, Jia H, Zhu M, Li X, Zhao S: Pseudorabies viral replication is inhibited by a novel target of miR-21. Virology 2014, 456-457,319-328. 
20. Manz, A.; Graber, N.; Widmer, H.M. Miniaturized total chemical analysis systems: A novel concept for chemical sensing. Sensors Actuat. B: Chem. 1990, 1, 244-248.

21. Fang, Z. Microfluidic Analysis Chip; First Edition.; Science Press: Beijing, 2003; ISBN 7-03-010872-8.

22. Wu, J.; Kodzius, R.; Xiao, K.; Qin, J.; Wen, W. Fast detection of genetic information by an optimized PCR in an interchangeable chip. Biomed. Microdevices 2012, 14, 179-186.

23. Zhou, C.; Mu, Y.; Yang, M.; Song, Q.; Zhang, Y.; Wu, Z.; Xiang, L.; Jin, W.; Jin, Q. A gravity-induced flow injection system for surface plasmon resonance biosensor. Talanta 2013, 112, 95-100.

24. Song, Q.; Gao, Y.; Zhu, Q.; Tian, Q.; Yu, B.; Song, B.; Xu, Y.; Yuan, M.; Ma, C.; Jin, W.; et al. A nanoliter self-priming compartmentalization chip for point-of-care digital PCR analysis. Biomed. Microdevices $2015,17,64$.

25. Nakanishi, H.; Kido, A.; Ohmori, T.; Takada, A.; Hara, M.; Adachi, N.; Saito, K. A novel method for the identification of saliva by detecting oral streptococci using PCR. Forensic Sci. Int. 2009, 183, 20-23.

26. Cardeñosa, D.; Quinlan, J.; Shea, K.H.; Chapman, D.D. Multiplex real-time PCR assay to detect illegal trade of CITES-listed shark species. Sci. Rep. 2018, 8, 1-10.

27. Moutailler, S.; Yousfi, L.; Mousson, L.; Devillers, E.; Vazeille, M.; Vega-Rúa, A.; Perrin, Y.; Jourdain, F.; Chandre, F.; Cannet, A.; et al. A New High-Throughput Tool to Screen Mosquito-Borne Viruses in Zika Virus Endemic/Epidemic Areas. Viruses 2019, 11, 904.

28. Song, Q.; Sun, J.; Mu, Y.; Xu, Y.; Zhu, Q.; Jin, Q. A new method for polydimethylsiloxane (PDMS) microfluidic chips to maintain vacuum-driven power using Parylene C. Sensors Actuat. B: Chem. 2017, 256, 1122-1130.

29. Quirasco, M.; Schoel, B.; Chhalliyil, P.; Fagan, J.; Gálvez, A. Real-time and conventional PCR detection of Liberty Link $B$ rice varieties and transgenic soy in rice sampled in the Mexican and American retail markets. Anal. Bioanal. Chem. 2008, 392, 395-404.

30. Xu, Y.; Cheng, W.; Chen, F. Application of Multiplex PCR for Detection of Food-borne Bacterial Pathogens. Food Science 2007, 02.

31. Zhong, K.; Tian, J.; Li, Y.; Liu, X.; Ji, R. Faster detection of Vibrio parahaemolyticus in foods by PCR technique. Chinese Journal of Food Hygiene 2004, 4.

32. Detection of bacterial $16 \mathrm{~S}$ ribosomal RNA genes for forensic identification of vaginal fluid. Leg. Med. 2012, 14, 160-162.

33. Gale, F. China's Pork Imports Rise Along with Production Costs; A Report from the Economic Research Service; United States Department of Agriculture, 2017; ISBN LDPM-271-01.

34. Vashist, S.K.; Luppa, P.B.; Yeo, L.Y.; Ozcan, A.; Luong, J.H.T. Emerging Technologies for NextGeneration Point-of-Care Testing. Trends Biotechnol. 2015, 33, 692-705.

35. Coelho, A.C.; García Díez, J. Biological Risks and Laboratory-Acquired Infections: A Reality That Cannot be Ignored in Health Biotechnology. Front. Bioeng. Biotechnol. 2015, 3.

36. Nagarajan K, Saikumar G. Fluorescent in-situ hybridization technique for the detection and localization of classical swine fever virus in infected tissues[J]. Veterinarski arhiv, 2012, 82(5): 495- 
504.

37. Yamazaki W, Mioulet V, Murray L, et al. Development and evaluation of multiplex RT-LAMP assays for rapid and sensitive detection of foot-and-mouth disease virus[J]. Journal of virological methods, 2013, 192(1-2): 18-24.

38. Alp A. Advancement in POCT molecular testing: the multiplex PCR POCT devices for infectious diseases[J]. EJIFCC, 2018, 29(3): 205.

39. Wu, J.; Cao, W.; Wen, W.; Chang, D.C.; Sheng, P. Polydimethylsiloxane microfluidic chip with integrated microheater and thermal sensor. Biomicrofluidics 2009, 3, 012005.

40. Tong, R.; Zhang, L.; Hu, C.; Chen, X.; Song, Q.; Lou, K.; Tang, X.; Chen, Y.; Gong, X.; Gao, Y.; et al. An Automated and Miniaturized Rotating-Disk Device for Rapid Nucleic Acid Extraction. Micromachines 2019, 10, 204.

41. Tong, R.; Zhang, L.; Song, Q.; Hu, C.; Chen, X.; Lou, K.; Gong, X.; Gao, Y.; Wen, W. A fully portable microchip real-time polymerase chain reaction for rapid detection of pathogen. Electrophoresis 2019, 40, 1699-1707.

\section{Tables}

Please see the supplementary files section to view the tables.

\section{Figures}

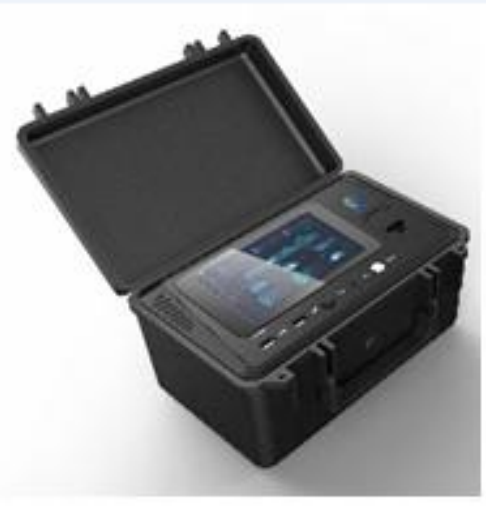

(A)

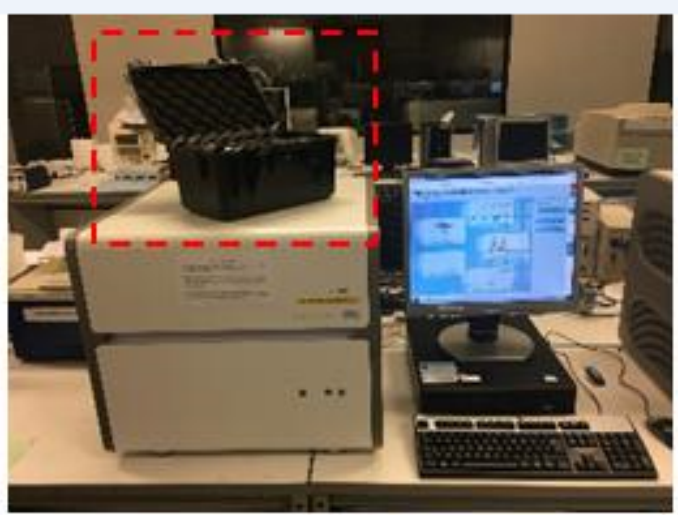

(B)

\section{Figure 1}

(a) The portable rapid microfluidic PCR system; (b) Size comparison of the microfluidic PCR instrument (within the red dotted box) with the traditional large PCR instrument (Roche 480). 

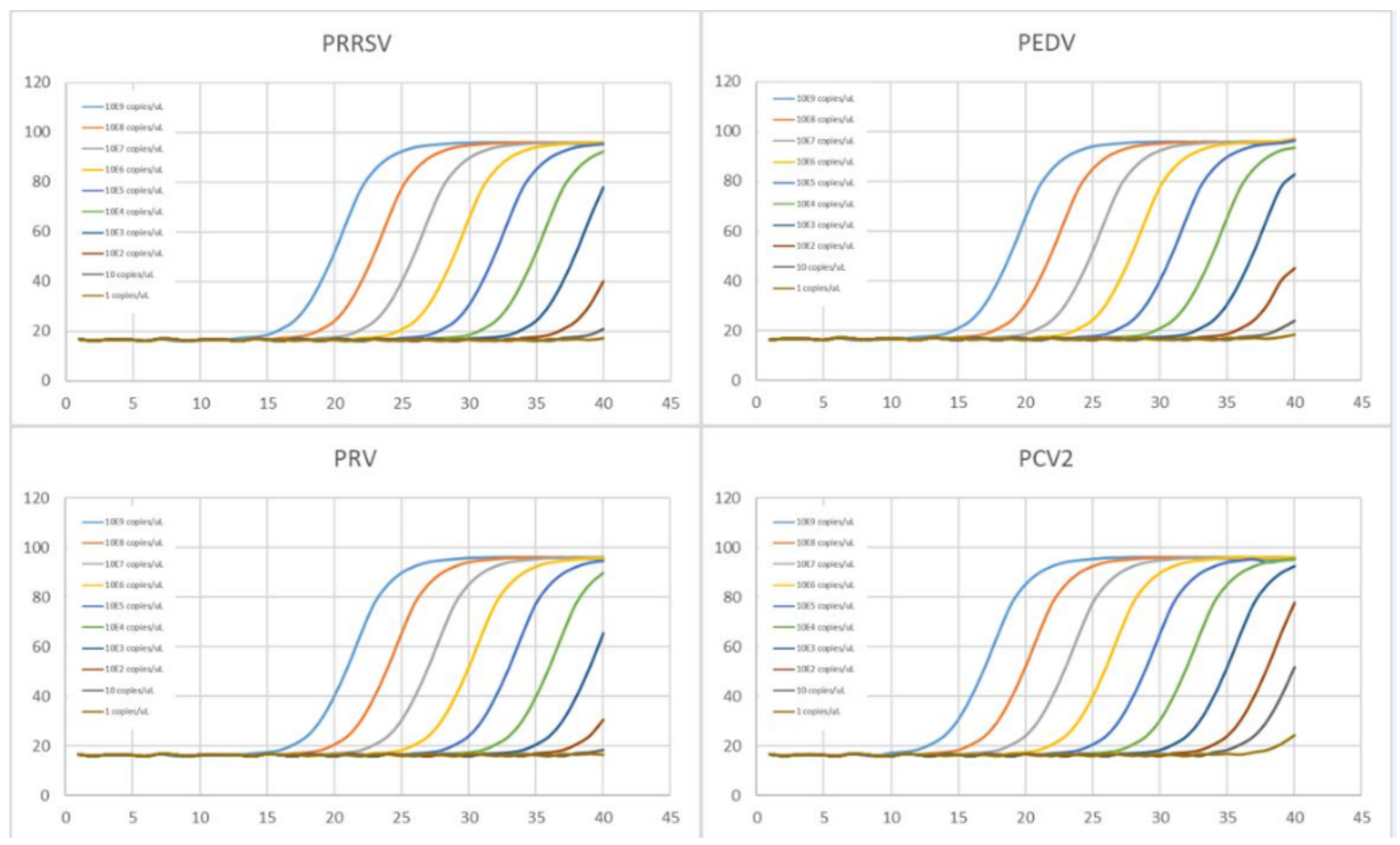

\section{Figure 2}

Sensitivity test of the PCR protocols of the four swine disease viruses. 

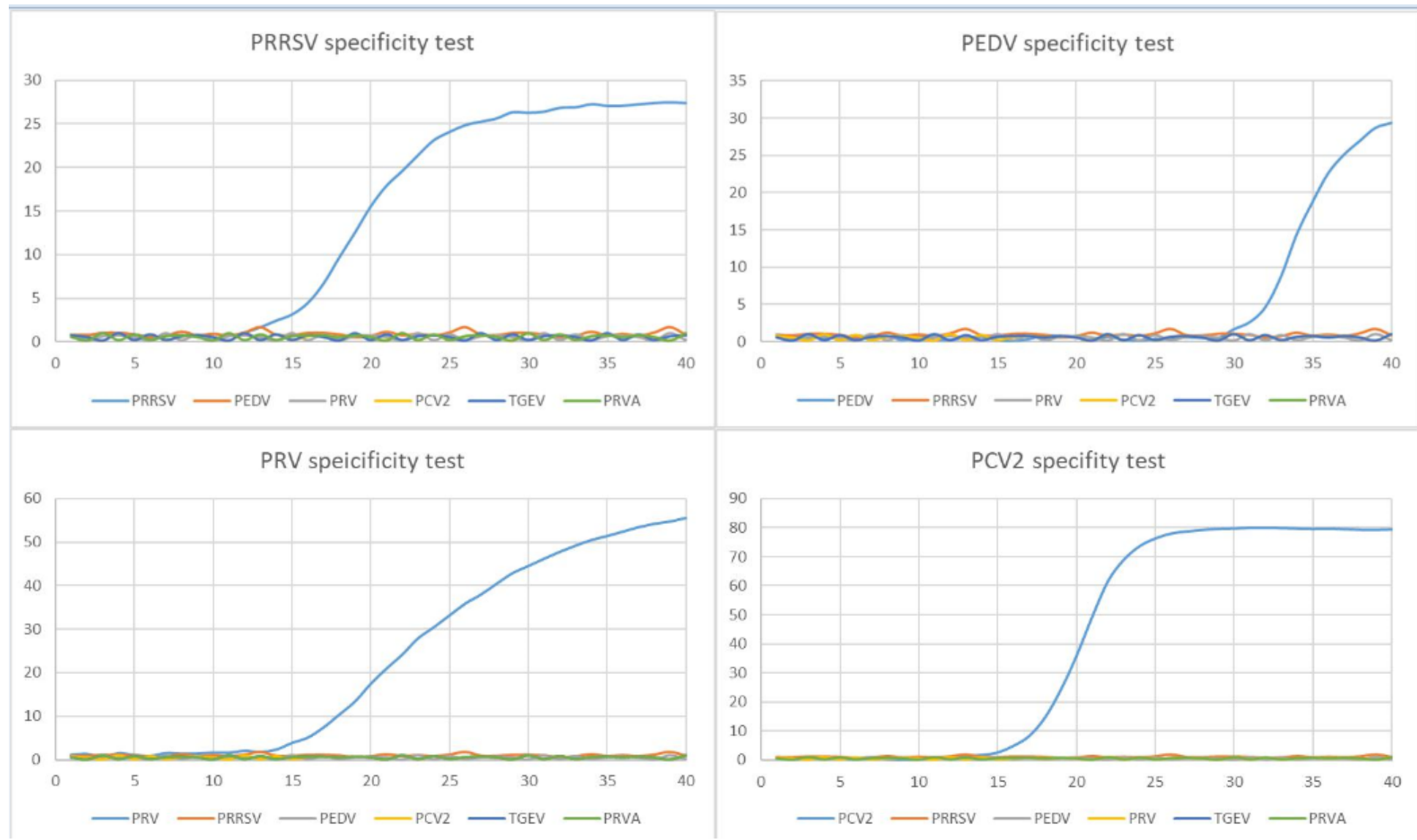

\section{Figure 3}

Specificity test of the four swine disease viruses on the chip.
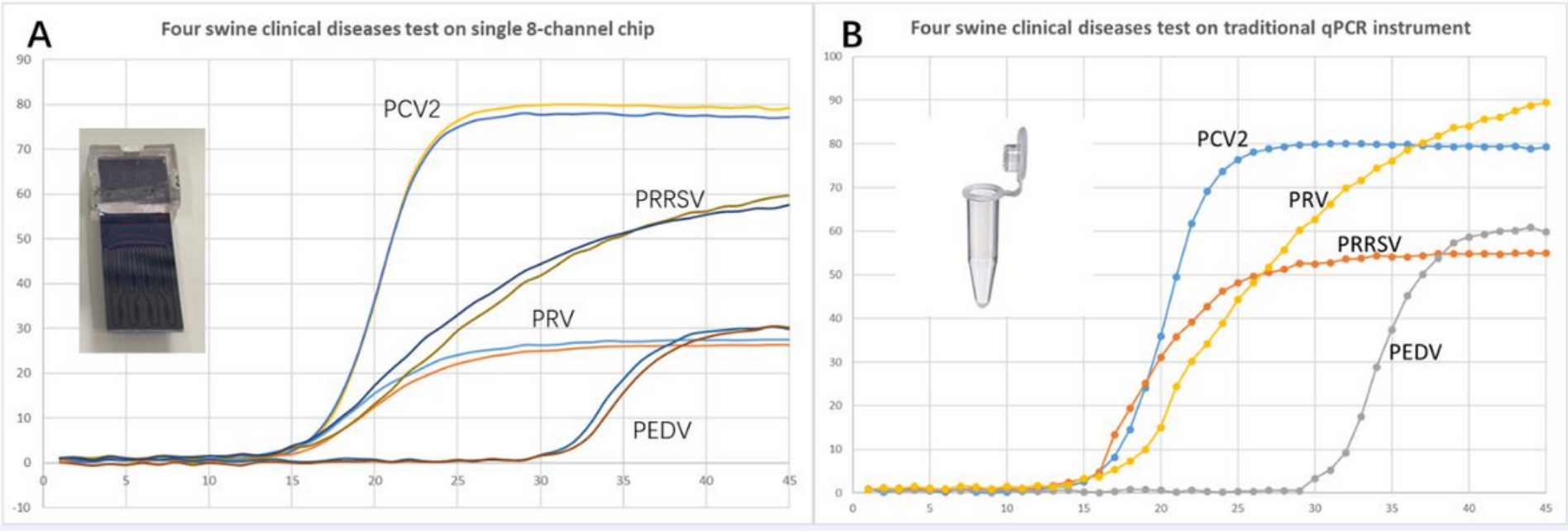

\section{Figure 4}

Parallel detection results of four clinical samples of swine diseases on the 8-chamber microfluidic chip (a) and on traditional tube-based PCR instrument (b) .

\section{Supplementary Files}


This is a list of supplementary files associated with this preprint. Click to download.

- Table1R.xlsx

- Table2.xlsx 